\title{
STUDY OF STAR FORMATION RATE AND METALLICITY OF AN INTERACTING DWARF GALAXY NGC 2604
}

\author{
Daya Nidhi Chhatkuli ${ }^{1 *}$, Sanjaya Paudel $^{2}$, Binil Aryal ${ }^{1}$ \\ ${ }^{1}$ Central Department of Physics, Tribhuvan University, Kirtipur, Kathmandu, Nepal, \\ ${ }^{2}$ Centre for Galaxy Evolution Research, Department of Astronomy, Yonsei University, Seoul, South Korea \\ *Corresponding author: chhatkulidn@gmail.com \\ (Received: June 02, 2020; Revised: October 13, 2020; Accepted: November 01, 2020)
}

\begin{abstract}
We present a study of the Sloan Digital all Sky Survey Data Release 12 (SDSS DR12) optical spectra of an interacting dwarf galaxy NGC 2604 that has redshift 0.0069 . Thirteen characteristic emission lines were identified in the wavelength range of $3885 \AA$ to $6742 \AA$, the strongest line was due to $\mathrm{H}_{\alpha}$ emission with a value of emission-line flux 1538.8 $\mathrm{erg} / \mathrm{s} / \mathrm{cm}^{2} / \AA$. The other twelve emission lines were observed because of OI doublet, $\mathrm{H}_{\beta}, \mathrm{H}_{\gamma}, \mathrm{H}_{\delta}$, OIII doublet, HeI, SII doublet and NII doublet transitions. Eleven characteristic lines agreed perfectly with the Gaussian distribution with greater than $99.9 \%$ coefficient of regression. However, full-width half maximum (FWHM) was found to be less than 5 $\AA$. No absorption metallic lines were observed in the spectra which indicates that the galaxy was either newly formed. The line metallicity of the galaxy was found to be 8.4 dex and the extinction coefficient was 0.2134 . The star formation rate due to $\mathrm{H}_{\alpha}$ emission after extinction correction was found to be $0.0927 \mathrm{M}_{\odot}$ year ${ }^{-1}$ which is almost double of the value $\left(0.057 \mathrm{M}_{\odot}\right.$ year $\left.^{-1}\right)$ before correction.
\end{abstract}

Keywords: Dwarf galaxy, Characteristic peaks, Extinction, $\mathrm{H}_{\alpha}$ line, Metallicity, Star formation rate.

\section{INTRODUCTION}

A small-sized galaxy, having low values of luminosity, surface brightness, and metallicity is known as a dwarf galaxy. According to Lambda Cold Dark Matter $(\Lambda \mathrm{CDM})$ cosmology, dwarf galaxies are expected to be the first formed dark matter dominated objects and large scale structures are built up by hierarchical merging of these objects. Karachentsev et al. (2004), reported that dwarf galaxies occupy more than $70 \%$ of all galaxies in the local Universe. Dwarf galaxies are the most abundant galaxies in the Universe but hard to be detected. The difficulties in detecting dwarf galaxies are mainly due to their low absolute luminosity and low surface brightness. Since only relatively brighter galaxies have been surveyed on large scale, the true number density of dwarf galaxies in the nearby Universe is poorly known. Davies and Morgan (1994) interpreted that the actual number density of dwarf galaxies in the nearby universe is poorly known because of the limited survey of relatively brighter galaxies only. The study of dwarf galaxies provides knowledge on the early process of structure formation and hence we can study the evolution of the Universe.

A dwarf galaxy is composed of as few as several thousand up to as high as a few billion stars. These dwarf galaxies often orbit around larger galaxies. For example, more than 20 dwarf galaxies are orbiting around our Milkyway galaxy (Noyola et al., 2008). Dwarf galaxies in the local universe are mainly classified into three categories. (i) Dwarf elliptical (dEs): The dwarfs that are gas-poor and have elliptical shapes are called dwarf elliptical galaxies. No evidences of recent star formation were found in such galaxies. Comparatively more spherical shaped dwarf galaxies found at the faint end of the dwarf elliptical scale are called spheroid (Sphs) $\left(\mathrm{Web}^{1}\right)$ (ii) dwarf irregulars (Irrs). The galaxies containing a considerable quantity of dust and gas which have clear evidence of star formation are called dwarf irregulars. (iii) star bursting dwarfs: The gas-rich galaxies having a high rate of star formation are called star bursting dwarfs. The starburst activity occurs in the galaxies having steeper gravitational potential. The starburst galaxies are gas-rich dwarf galaxies with high dark matter content, low mass, and low chemical abundance. Most of the dwarf galaxies were formed by the interaction of galaxies. Okazaki and Taniguchi (2000) analyzed that gas-rich galaxies were formed by merger or collision of the galaxies while gas-poor elliptical galaxies was found at the center of galaxy clusters.

The galaxies with low mass, low chemical abundances, high gas, and dark matter content were termed as starforming dwarf galaxies. Such galaxies mostly reside in the field environment. The star formation rate (SFR) of a galaxy can be obtained in various wavelength regions like $\mathrm{X}$-ray, ultraviolet, optical, far infrared etc. by making use of the experimental/observational calculations obtained from samples of the above-mentioned galaxy types: elliptical, irregulars, or starbursts. The star formation rate of a galaxy depends upon different factors like interstellar gases, temperature, molecular cloud of neutral hydrogen etc. (Thronson \& Telesco, 1986). Similarly, metallicity is also one of the factors. The star formation rates in the low metallic dwarf galaxies were found to be overestimated by a factor greater than or equal to 3 (Weilbacher et al., 2001). 
In this paper, the spectroscopic analysis was performed on SDSS DR12 spectra of low red-shifted dwarf galaxy NGC 2604. The characteristic peaks of the emission lines were identified along with their responsible elements for the emission. The star formation rate (SFR), extinction coefficient and emission line metallicity were also calculated using Gaussian fits.

\section{Region of interest}

We selected a system of interacting dwarf galaxy NGC 2604 having a low redshift of $\mathrm{z}=0.0069$ from the catalog compiled by Poudel et al. (2018). The galaxy NGC 2604 is an interacting galaxy located at R.A. $($ J2000) $=$ $128.3457^{0}$, Dec. $(\mathrm{J} 2000)=29.5386^{\circ}$ (Fig. 1a). The SDSS photometric $\mathrm{u}, \mathrm{g}, \mathrm{r}, \mathrm{i}$, and $\mathrm{z}$ color magnitudes are respectively, $14.41 \mathrm{mag}, 13.89 \mathrm{mag}, 13.36 \mathrm{mag}, 13.36$ and 13.12 mag. $\left(\mathrm{Web}^{2}\right)$. NGC 2604 is the barred-spiral galaxy that can be observed from Kathmandu, Nepal if viewed from a telescope, in the evening around 19:09 (+0545), $69^{\circ}$ above the eastern horizon. It reaches its highest point in the sky at 20:40, $88^{\circ}$ above the northern horizon from Kathmandu, and will continue to appear till around 02:00 $\left(\mathrm{Web}^{3}\right)$.

An emerging concept regarding the barred galaxies is that the bars play a controlling role in the comprehensive star formation rate of the disk galaxies. Tubbs (1982) proposed the probability of a decrease in total starforming rates due to bars in the galaxy. Kim et al. (2017) showed that there is a significantly lower star-forming activity of strongly-barred galaxies than their unbarred counterparts. The SDSS optical image of the galaxy UGC 2604 is shown in Fig. 1(a) which is interacting with a nearby companion NGC 2604B. We can see clearly from the image that it is a barred type galaxy. The spectrum of the galaxy is shown in Fig. 1(b). In the spectra, several characteristic lines due to different elements are found. The most prominent line is the $\mathrm{H}_{\alpha}$ line which corresponds to $6564.4 \AA$. Then the intensity goes on decreasing with $\mathrm{H}_{\beta}$, OIII, NII, $\mathrm{H}_{\gamma}$ lines, and so on. The characteristic lines will be studied in this paper and the reason behind those emissions in the dwarf galaxy will be investigated.

The main objective of this work is the spectroscopic analysis of the galaxy UGC 2604. The specific objective is to calculate the star formation rate of the galaxy from the $\mathrm{H}_{\alpha}$ line, calculate line ratio, metallicity, extinction coefficient and compare the star formation rate.

\section{MATERIALS AND METHODS}

This study was based on the Sloan Digital Sky Survey (SDSS) database (http://www:sdss.org/collaboration/ credits.html). The SDSS DR12 spectral data was extracted from software called ALADIN. The data was plotted using the software ORIGIN 8.0 to obtain spectra of the galaxy. The peaks in emission lines were identified and performed the Gaussian fits on the spectra of emission lines. It was assumed that the characteristic lines get broaden because of Doppler broadening, permitted line cooling, and fine structure cooling. These are natural processes; therefore the Gaussian distribution was expected.

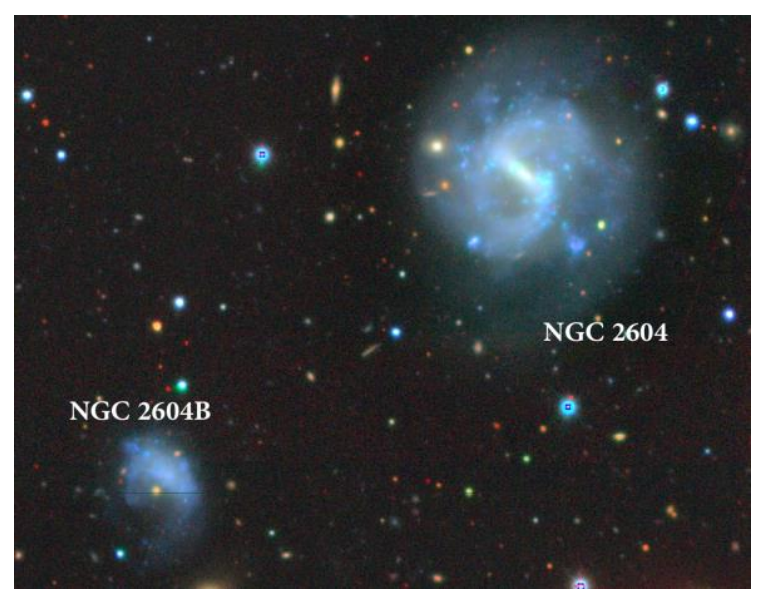

(a)

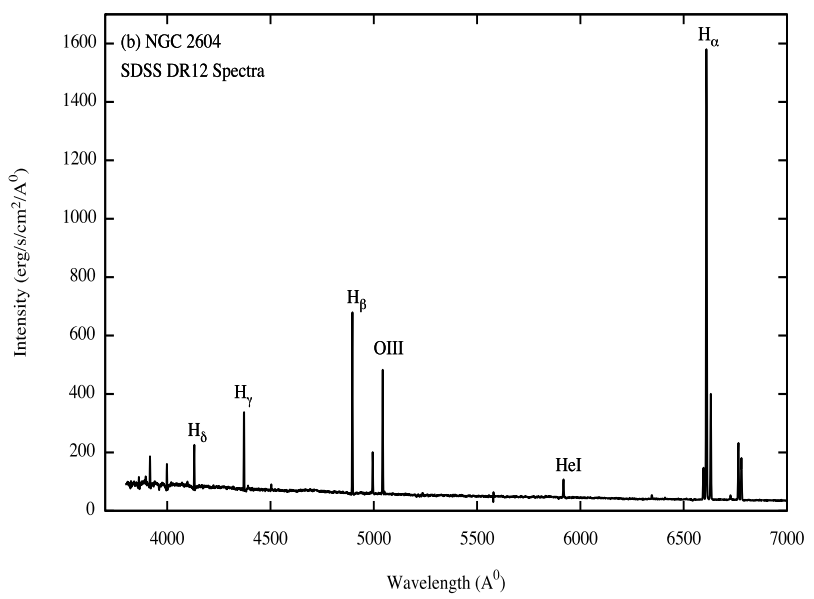

(b)

Fig. 1. (a) SDSS image of dwarf galaxy [Source: SDSS DR12 Science Archive Server], (b) SDSS DR12 spectra of NGC 2604

Mathematically Gaussian distribution (Squires, 2001) is defined as;

$$
f_{G}(x)=\frac{1}{\sqrt{2 \pi \sigma^{2}}} e^{\frac{-(x-\mu) 2}{2 \sigma^{2}}}
$$

Where, $x$ is a normal random variable, $\mu$ is the mean and $\sigma$ is the standard deviation of the distribution. If the number of events is very large, then the Gaussian distribution function may be used to describe physical events. The Gaussian distribution is a continuous function that approximates the exact binomial distribution of events. Any deviation from the Gaussian distribution will be analyzed critically. Jha et al. (2017) studied the variation of flux density with density of a structure of size $4.46 \mathrm{pc} \times$ 
$2.36 \mathrm{pc}$ and found a cavity structure at $100 \mu \mathrm{m}$ images in interstellar medium. From the study of distribution of flux density with distance along major diameter of the structure, they found that the distribution strongly deviated from the Gaussian distribution. This is due to the pulsar wind during the formation of the cavity in interstellar medium.

A table of Gaussian parameters for noticeable characteristic peaks was presented. In the spectra (Fig. 1b), 81 peaks were noticed above the continuum. Out of them, only 13 characteristic peaks having maximum intensity were presented here along with the responsible elements and their Gaussian parameters. Finally, $\mathrm{H}_{\alpha}$-line was identified, and using the Gaussian area for intensity of the line, star formation rate (SFR) in the dwarf galaxy NGC 2604 was calculated. The star formation rate was calculated by adopting the empirical formula proposed by Kennicutt (1998) as follows;

$\operatorname{SFR}\left(\mathrm{M}_{\odot}\right.$ year $\left.^{-1}\right)=7.9 \times 10^{-42} \sum \mathrm{L}\left(\mathrm{H}_{\alpha}\right) \quad\left(\operatorname{ergs~s}^{-1}\right)$

Here, $\sum \mathrm{L}\left(\mathrm{H}_{\alpha}\right)$ is the total luminosity of $\mathrm{H}_{\alpha}$ which was calculated by using Gaussian fits. $\sum \mathrm{L}\left(\mathrm{H}_{\alpha}\right)=$ Area of Gaussian fit $\times 10^{-17} \times 4 \pi \mathrm{R}^{2}$ (ergs s${ }^{-1}$ ). $\mathrm{R}$ is the radius of the sphere which was calculated as $\mathrm{R}=\mathrm{D} \times 3.08 \times 10^{24} \mathrm{~cm}$. Here, $\mathrm{D}$ is luminosity distance in Mpc. From the calibration provided by Marino et al. (2013), the emission line metallicity using a line ratio between NII and $\mathrm{H}_{\alpha}$ was calculated by

$12+\log (\mathrm{O} / \mathrm{H})=8.743+0.462 \times \log \left(\mathrm{NII} / \mathrm{H}_{\alpha}\right)$

\section{RESULTS AND DISCUSSION}

Out of thirteen emission lines selected, only twelve characteristic emission lines are shown in Figs. 2 and 3.The Gaussian fits of the first six emission lines of HeI, $\mathrm{H}_{\delta}, \mathrm{H}_{\gamma}, \mathrm{H}_{\beta}$, OIII, and OIII are shown in Fig. 2. Solid curves represent Gaussian fits. The statistical error bars $( \pm 1 \sigma)$ were shown. The broadening of the characteristic line was mainly because of the Doppler broadening. The wavelengths given in the $\mathrm{X}$-axis were redshift corrected. A conservative estimate of the flux error is shown in the plot, i.e., $10 \%$ of the observed flux provided by the SDSS webpage (https://www.sdss.org/dr15/spectro/caveats/).

Likewise, the Gaussian fits of the second six emission lines of OI, NII, $\mathrm{H}_{\alpha}$, NII, SII and SII are shown in Fig. 3. Here also, solid curves represented Gaussian fits. The statistical error bars $( \pm 1 \sigma)$ are shown. The broadening of the characteristic line was mainly because of the Doppler broadening.

A very good agreement between the observed distribution and Gaussian fit was found, as shown in Figs. 2 and 3. The lines $\mathrm{H}_{\delta}, \mathrm{H}_{\gamma}, \mathrm{H}_{\beta}$, and $\mathrm{H}_{\alpha}$ are Balmer lines. The corrected and calibrated Gaussian area was used to find the values of the star formation rate. The Gaussian fits shown in Figs. 2 and 3 ranged from $3885 \AA$ to $6742 \AA$ in the spectra.
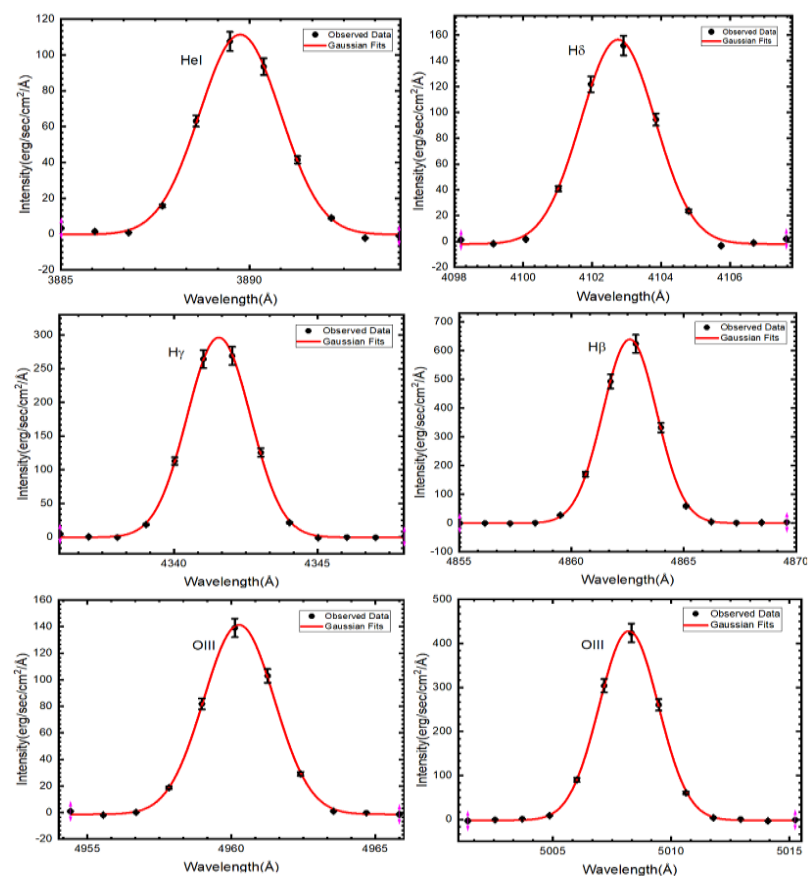

Fig. 2. First six characteristic peaks of the SDSS DR12 spectra of interacting dwarf galaxy NGC 2604 in the range $3885 \AA$ to $5015 \AA$
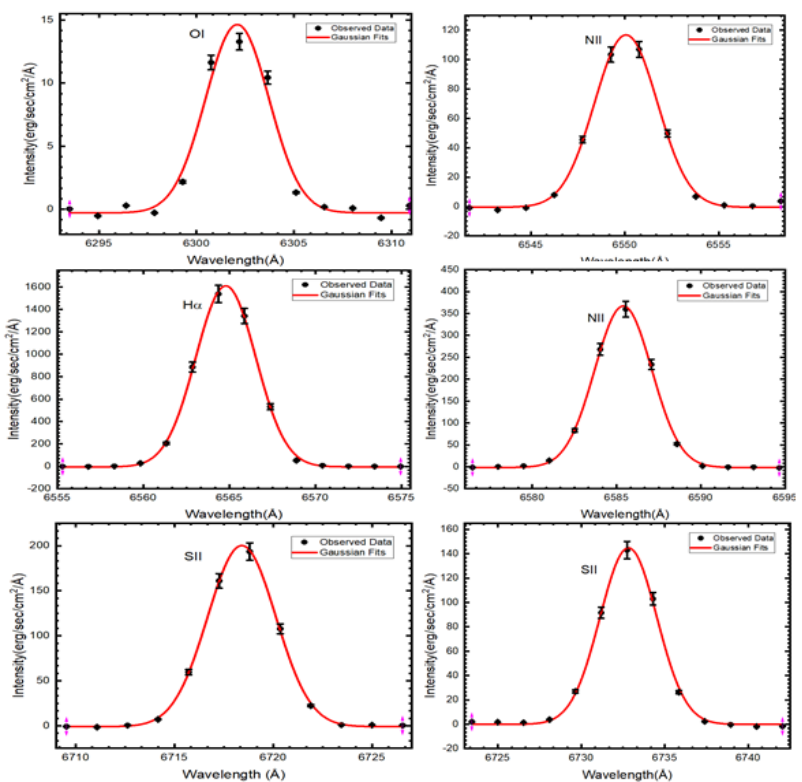

Fig. 3. Second six characteristic peaks of the SDSS DR12 spectra of interacting dwarf galaxy NGC 2604 in the range $6293 \AA$ to $6742 \AA$. Error bars represent the $\pm 1 \sigma$ statistical errors

Table 1 shows a list of Gaussian parameters of 13 strongest peaks (shown in Fig. 2 and 3 ) in the SDSS 
spectra of dwarf galaxy NGC 2604. The first, second, third, and fourth columns respectively represent the name of the responsible element for the emission, the peak wavelength $\left(\lambda_{p}\right)$, the value of peak intensity $\left(I_{p}\right)$ in $\mathrm{erg} / \mathrm{s} / \mathrm{cm}^{2} / \AA$ corresponding to peak wavelength and full width half maximum (FWHM). Similarly, the fifth, sixth, seventh, and eighth columns represent area, height of Gaussian curve, offset, and coefficient of regression of the fitted data, respectively, as summarized in Table 1.

Gaussian offset which showed the skewness of the data is the positional difference between the Gaussian peak and the observed peak in the spectra. The coefficient of regression is represented by $R^{2}$ which was found to be more than 0.990 except for OI, suggesting a very good agreement with Gaussian distribution. The small values (2.53 - 4.10) $\AA$ of full-width half maxima (FWHM) represent the characteristic peak. Interestingly, the characteristic peaks showed Gaussian nature. The intensity was found to be maximum $\left(1538.8 \mathrm{erg} / \mathrm{s} / \mathrm{cm}^{2} / \mathrm{A}\right)$ at the wavelength of $6564.4 \AA$. This line was because of $\mathrm{H}_{\alpha}$ emission. The second strongest line was due to $\mathrm{H}_{\beta}$ which was found at the wavelength of $4862.9 \AA$ with peak intensity $624.0 \mathrm{erg} / \mathrm{s} / \mathrm{cm}^{2} / \AA$. The weakest intensity among the 13 strongest lines was observed at the wavelength of $6364.9 \AA$ with peak intensity $5.3 \mathrm{erg} / \mathrm{s} / \mathrm{cm}^{2} / \AA$, which was due to neutral oxygen OI.

Table 1. Description of the selected characteristic emission lines of spectra of dwarf galaxy NGC 2604 shown in Fig. 1

\begin{tabular}{cccccccc}
\hline Element & $\lambda_{\mathrm{p}}(\AA)$ & $\mathrm{I}_{\mathrm{p}}$ & FWHM $(\AA)$ & Area $\left(\mathrm{erg} / \mathrm{s} / \mathrm{cm}^{2} / \AA\right)$ & Height $\left(\mathrm{erg} / \mathrm{s} / \mathrm{cm}^{2} / \AA\right)$ & Offset & $\mathrm{R}^{2}$ \\
\hline $\mathrm{HeI}$ & 3889.5 & 107.5 & 2.53 & 300.60 & 111.53 & +0.269 & 0.998 \\
$\mathrm{H}_{\delta,}$ & 4102.9 & 151.9 & 2.54 & 428.33 & 158.49 & +0.158 & 0.997 \\
$\mathrm{H}_{\gamma}$ & 4342.0 & 269.0 & 2.60 & 820.17 & 296.62 & +0.465 & 0.999 \\
$\mathrm{H}_{\beta}$ & 4862.9 & 624.0 & 2.82 & 1920.98 & 638.95 & +0.267 & 0.999 \\
$\mathrm{OIII}$ & 4960.1 & 139.1 & 2.89 & 439.30 & 142.61 & -0.149 & 0.999 \\
$\mathrm{OIII}$ & 5008.3 & 423.5 & 2.95 & 1349.96 & 430.02 & +0.109 & 0.999 \\
$\mathrm{OI}$ & 6302.2 & 13.3 & 3.83 & 60.77 & 14.91 & +0.109 & 0.973 \\
$\mathrm{OI}$ & 6364.9 & 5.3 & 3.76 & 23.65 & 5.91 & -0.880 & 0.925 \\
$\mathrm{NII}$ & 6550.8 & 106.9 & 3.95 & 492.02 & 117.16 & +0.709 & 0.998 \\
$\mathrm{H}$ & 6564.4 & 1538.8 & 4.10 & 7048.21 & 1615.05 & -0.431 & 0.999 \\
$\mathrm{NII}$ & 6585.6 & 359.7 & 3.99 & 1563.03 & 368.36 & +0.151 & 0.999 \\
$\mathrm{SII}$ & 6718.8 & 193.5 & 4.07 & 871.40 & 201.16 & +0.404 & 0.999 \\
$\mathrm{SII}$ & 6732.7 & 142.9 & 4.01 & 619.02 & 144.89 & -0.082 & 0.998 \\
\hline
\end{tabular}

It can be seen from Table 1 that all the selected thirteen spectral Gaussian fits corresponding to the elements HeI, $\mathrm{H}_{\delta}, \mathrm{H}_{\gamma}, \mathrm{H}_{\beta}$, OIII, OI, NII, NII, and SII have positive values of offset which indicates that they have positive skewness. This means that the tail on the R.H.S. was longer than that on the L.H.S. on the curve and the mode was less than the mean. The rest of the Gaussian fits corresponding to OIII, OI, $\mathrm{H}_{\alpha}$, and SII lines have negative values of offset, indicating that they have negative skewness. It means that the tail on the L.H.S. of the curve was longer than the tail on the R.H.S. and the mode was more than the mean. The line due to $\mathrm{H}_{\alpha}$ has maximum FWHM (i.e., $4.10 \AA$ ), indicating that it was in very good agreement with Gaussian fit.

From Table 1, it can be seen that corresponding to the wavelength $6564.4 \AA$, maximum intensity of 1538.8 $\mathrm{erg} / \mathrm{sec} / \mathrm{cm}^{2} / \AA$ was observed in the spectral line due to $\mathrm{H}_{\alpha}$. The wavelength of the $\mathrm{H}_{\alpha}$ spectral line ranged from $6555.29 \AA$ to $6574.94 \AA$. Therefore, the maximum Gaussian area of the line with $7048.21 \mathrm{erg} / \mathrm{s} / \mathrm{cm}^{2} / \AA$ in the unit of intensity was detected. This might affect the star formation rate in the Galaxy. Statistically, $\mathrm{R}^{2}$ measures how close the data are to the fitted curve. The value of $R^{2}$ in all the spectral lines was almost $100 \%$. This fact also suggests that the fitted data is Gaussian-like.

\section{Star formation rate}

Area of Gaussian curve for $\mathrm{H}_{\alpha}$ line $=7048.21\left(10^{-17}\right.$ $\left.\mathrm{erg} / \mathrm{s} / \mathrm{cm}^{2} / \AA\right)$. For $\mathrm{H}_{\alpha}$ line, the intensity was maximum. Using the adopted distance to the galaxy $\mathrm{D}=29.29 \mathrm{Mpc}$ (Poudel et al., 2018), we calculated $\mathrm{R}=\mathrm{D} \times 3.08 \times 10^{24}$ $\mathrm{cm}=29.29 \times 3.08 \times 10^{24} \mathrm{~cm}=90.21 \times 10^{24} \mathrm{~cm}$. Star formation rate using $\mathrm{H}_{\alpha}$ line was calculated by using equation (2).

SFR $=7.9 \times 10^{-42} \times$ area of Gaussian fit $\times 10^{-17} \times 4 \pi \mathrm{R}^{2}=7.9 \times$ $10^{-42} \times 7048.21 \times 10^{-17} \times 4 \pi\left(90.21 \times 10^{24}\right)^{2}=0.057 \mathrm{M}_{\odot}$ year $^{-1}$

Line ratio $\mathrm{H}_{\alpha} / \mathrm{H}_{\beta}=7048.21 / 1920.98=3.67$. This is slightly greater than the theoretical value (2.8), which is due to internal extinction. 
The current star formation rate in our Milky Way galaxy was 2.6, the average star formation rate was about 8.25 $\mathrm{M}_{\odot}$ year $^{-1}$ (Kalberla \& Kerp, 2009). GALEX mission revealed that the star formation rate in the early-type galaxies, dwarf galaxies, low surface brightness galaxies, and the extreme outer disks of many normal galaxies was rather low. Although star formation had been detected occasionally in nearby elliptical and S0 galaxies (e.g., Pogge \& Eskridge, 1993), most of these galaxies have historically been regarded as "dead" in terms of recent star formation. Kennicutt et al. (2008) compiled $\mathrm{H}_{\alpha}$ luminosities for galaxies within the local $11 \mathrm{Mpc}$, which included $~ 300$ dwarf galaxies $\left(M_{B}>-17\right)$. Excluding a handful of dwarf spheroidal galaxies that have no cold gas, only 10 of these $(\sim 3 \%)$ were not detected in $\mathrm{H}_{\alpha}$, meaning that star formation had taken place over the last 3-5 Myr in the other $97 \%$ of the systems. Therefore, our dwarf galaxy meets this criterion.

\section{Metallicity}

The line ratio of NII $\left(\lambda_{\text {peak }}=6585.6 \AA\right)$ with $\mathrm{H}_{\alpha}$ was calculated as, $\mathrm{NII} / \mathrm{H}_{\alpha}=1563.03 / 7048.21=0.2218$. Line metallicity was $12+\log (\mathrm{O} / \mathrm{H})=8.743+0.462 \times \log$ $\left(\mathrm{NII} / \mathrm{H}_{\alpha}\right)=8.743+0.462 \times \log (0.2218)=8.44 \mathrm{dex}$ which is slightly lower than solar value.

\section{Extinction correction}

The line ratio $\mathrm{H}_{\alpha} / \mathrm{H}_{\beta}$ which is also called Blamer decrement is given by, $\mathrm{c}=3.67$. The theoretical value of $\mathrm{c}_{0}=2.86$ for an electron temperature of $10^{4} \mathrm{~K}$. Now extinction coefficient $\mathrm{E}(\mathrm{B}-\mathrm{V})=1.97 \times \log \left(\mathrm{c} / \mathrm{c}_{0}\right)=1.97 \times$ $\log (3.67 / 2.86)=0.2134$. Now, extinction $\mathrm{A}(\mathrm{H} \alpha)=2.45$ $\mathrm{E}(\mathrm{B}-\mathrm{V})=2.45 \times 0.2134=0.5228$.

Again, we have $\mathrm{A}(\mathrm{H} \alpha)=-2.5 \log (\mathrm{F}(\mathrm{H} \alpha) \mathrm{obs} / \mathrm{F}(\mathrm{H} \alpha) \mathrm{em})$. Using the value of observed flux $\mathrm{F}(\mathrm{H} \alpha)$ obs $=7048.21$ in this equation, the emitted flux was calculated to be $\mathrm{F}(\mathrm{H} \alpha) \mathrm{em}=11481.54 \mathrm{erg} / \mathrm{s} / \mathrm{cm}^{2} / \mathrm{A}$. Thus, using this value, the star formation rate was calculated as,

Star formation rate due to $\mathrm{H}_{\alpha}$ line $=7.9 \times 10^{-42} \times$ Area of Gaussian fit $\times 10^{-17} \times 4 \pi \mathrm{R}^{2}=7.9 \times 10^{-42} \times 11481.54 \times 10^{-17}$ $\times 4 \pi\left(90.21 \times 10^{24}\right)^{2}=0.0927 \mathrm{M}_{\odot}$ year $^{-1}$

Thus the SFR of the Galaxy before extinction was 0.057 $\mathrm{M}_{\odot}$ year $^{-1}$ and after extinction correction was $0.0927 \mathrm{M}_{\odot}$ year $^{-1}$. We can see that the SFR of the galaxy calculated after extinction correction is more (almost double) than that before correction indicating that the actual value of the flux of the galaxy is almost double of the observed value of the flux. This galaxy NGC 2604 has only three low mass galaxies around it which means that the galaxy is almost in the field environment.

\section{CONCLUSION}

We studied SDSS DR12 emission spectra of an interacting dwarf galaxy NGC 2604 located at R.A.
$(\mathrm{J} 2000)=128.3457^{\circ}$, Dec. $(\mathrm{J} 2000)=29.5386^{\circ}$, having redshift 0.0069 . We noticed thirteen significant peaks from Gaussian fits. Present results conclude as follows:

1. The strongest emission line was found for $\mathrm{H}_{\alpha}$ with intensity $1538.8 \mathrm{erg} / \mathrm{s} / \mathrm{cm}^{2} / \AA$. The line ratio $\mathrm{H}_{\alpha} / \mathrm{H}_{\beta}$ was found to be 3.67

2. Other emission lines were observed because of neutral Oxygen (OI), Balmer lines $\mathrm{H}_{\delta}, \mathrm{H}_{\gamma}, \mathrm{H}_{\beta}$, doubly ionized oxygen (OIII) doublet, neutral Helium (HeI), singly ionized Sulpher (SII) doublet, and singly ionized nitrogen (NII) doublet.

3. All the eleven characteristic lines showed a very good agreement with Gaussian fits greater than 99.9 $\%$ coefficient of regression. However, full width at half maxima (FWHM) was found to be less than 5 $\AA$. These lines were broadened because of Doppler broadening and permitted line cooling.

4. No metal absorption line was observed in the spectra of interacting dwarf galaxy NGC 2604. This suggests that the galaxy was either newly formed.

5. Star formation rate (SFR) due to $\mathrm{H}_{\alpha}$ emission line was found to be $0.057 \mathrm{M}_{\odot}$ year $^{-1}$ which is considered as a low star formation rate.

\section{ACKNOWLEDGEMENTS}

One of the authors, Daya Nidhi Chhatkuli acknowledges the University Grants Commission of Nepal for financial support by providing a Ph.D. Fellowship (PhD-75/76-S \& T-13) to carry out the Ph.D. works.

\section{REFERENCES}

Davies, J., \& Morgan, I. (1994). Why are dwarf galaxies so difficult to find? European Southern Observatory Conference and Workshop Proceedings, 49, 19-26.

Jha, A. K., \& Aryal, B. (2017). A study of a pulsar wind driven structure in far-infrared IRAS map at latitude$10^{\circ}$. Journal of Institute of Science and Technology, 22(1), 1-9.

Kalberla, P. M., \& Kerp, J. (2009). The H1 distribution of the milkyway. Annual Review of Astronomy and Astrophysics, 47, 27-61.

Karachentsev, I. D., Karachentseva, V. E., Huchtmeier, W. K., \& Makarov, D. I. (2004). A catalog of neighboring galaxies. The Astronomical Journal, 127(4), 2031-2068.

Kennicutt Jr, R. C. (1998). Star formation in galaxies along the hubble sequence. Annual Review of Astronomy and Astrophysics, 36(1), 189-231.

Kennicutt Jr, R. C., Lee, J. C., Funes, J. G., Sakai, S., \& Akiyama, S. (2008). A H $\alpha$ imaging survey of galaxies in the local $11 \mathrm{Mpc}$ volume. The 
Astrophysical Journal Supplement Series, 178(2), 247-279.

Kim, E., Hwang, H. S., Chung, H., Lee, G. H., Park, C., Sodi, B. C., \& Kim, S. S. (2017). Star formation activity of barred spiral galaxies. The Astrophysical Journal, 845(2), 1-12.

Marino, R. A., Rosales-Ortega, F. F., Sánchez, S. F., De Paz, A. G., Vílchez, J., Miralles-Caballero, D., ... \& Díaz, A. I. (2013). The $\mathrm{O}_{3} \mathrm{~N}_{2}$ and $\mathrm{N}_{2}$ abundance indicators revisited: improved calibrations based on CALIFA and Te-based literature data. Astronomy \& Astrophysics, $\quad 559$, https://doi.org/10.1051/0004-6361/201321956

Noyola, E., Gebhardt, K., \& Bergmann, M. (2008). Gemini and hubble space telescope evidence for an intermediate-Mass black hole in $\omega$ centauri. The Astrophysical Journal, 676(2), 1008-1015.

Okazaki, T., \& Taniguchi, Y. (2000). Dwarf galaxy formation induced by galaxy interactions. The Astrophysical Journal, 543(1), 149-152.

Pogge, R. W., \& Eskridge, P. B. (1993). Star formation in the disks of HI-rich S0 galaxies. The Astronomical Journal, 106, 1405-1419.
Poudel, S., Smith, R., Yoon, S. J., Calderón-Castillo, P., \& Duc, P. A. (2018). A catalog of merging dwarf galaxies in the local universe. The Astrophysical Journal Supplement Series, 237(2), 1-20.

Squires, G. L., (2001). Practical physics. Cambridge University Press.

Thronson Jr, H. A., \& Telesco, C. M. (1986). Star formation in active dwarf galaxies. The Astrophysical Journal, 311, 98-112.

Tubbs, A. D. (1982). The inhibition of star formation in barred spiral galaxies. The Astrophysical Journal, $255,458-466$.

Web ${ }^{1}$ http://astronomy.swin.edu.au/cosmos/D/Dwarf+Gala xy.

Web ${ }^{2}$ http://skyserver.sdss.org/dr9/en/tools/quicklook/quic kobj.asp?id=1237660765373988975.

Web ${ }^{3}$ https://in-the-sky.org/data/object.php?id=NGC2604 .

Weilbacher, P. M. (2001). On star formation rates in dwarf galaxies. Astronomy \& Astrophysics, 373(1), L9-L12. 